\title{
Pulmonary rehabilitation referral and uptake from primary care for people living with COPD: a mixed-methods study
}

\section{To the Editor:}

Pulmonary rehabilitation (PR) for people with COPD leads to clinically significant improvements in quality of life and exercise capacity [1]. In England and Wales, UK, in 2013-2014, only 15\% of eligible patients were referred (51\% from primary care), of whom $31 \%$ did not attend assessment [2]. We aimed to generate a theory-informed understanding of enablers and barriers to PR referral and uptake from primary care.

This mixed-methods study [3] used normalisation process theory (NPT) [4] to understand how PR referral becomes embedded in clinical practice via NPT constructs of coherence (how clinicians understand and value PR), cognitive participation (how PR referral is sustained in practice), collective action (how PR referral is operationalised) and reflexive monitoring (how clinicians judge whether PR referral is worthwhile). Burden of treatment theory (BoT) [5] informed understanding of the patient experience via parallel constructs relating to how patients make sense of their condition and treatment, how they organise self-care work, what taking up PR involves for patients and how patients judge if PR is worthwhile.

Online surveys (dichotomous, multiple response, Likert scales and free-text items) were sent to all PR providers in the East of England (EoE), to other PR providers in England and Wales who contributed to the national COPD audit, and to all primary care practices in the EoE. Purposive sampling was used to recruit for semistructured interviews and focus groups, targeting all EoE PR providers and, in EoE areas with higher and lower PR utilisation rates, general practitioners (GPs), practice nurses, commissioners and patients (white British and South Asian) who had accepted a PR offer, declined PR or were not referred. Survey, interview and focus group questions were mapped to NPT constructs. Qualitative data and free-text survey responses were analysed following a framework approach [6] informed by NPT constructs. Qualitative data collection and concurrent analysis continued until saturation was achieved, i.e. deductive codes were adequately represented and no new codes identified [7]. Quantitative data were analysed descriptively. GP and nurse survey responses were compared using Chi-squared analysis. Research questions and interview/focus group guides were reviewed by the patient and participant involvement group.

Informed consent was obtained from all participants. The study was approved by Cambridge Central Research Ethics Committee (ref. 17/EE/0136).

EoE primary care practices (107 out of $415,25.8 \%$ ), EoE PR services (14 out of $20,70 \%$ ), and other England and Wales PR services (46 out of 179, 25.7\%) completed the surveys. 32 clinicians, six commissioners and 42 COPD patients took part in interviews and focus groups. 28 patients had accepted a PR offer, seven had declined, seven were not referred and seven were of South Asian heritage (table 1).

From the survey and qualitative research, we identified enablers and barriers across six domains (figure 1).

$@$ ERSpublications

Healthcare service and patient barriers contribute to low referral to and uptake of pulmonary rehabilitation (PR). Solutions should support skilled clinician-patient conversations and span primary care-PR boundaries to prevent disjointed working. http://bit.ly/2PVKHZf

Cite this article as: Early F, Wilson PM, Deaton C, et al. Pulmonary rehabilitation referral and uptake from primary care for people living with COPD: a mixed-methods study. ERJ Open Res 2020; 6: 00219-2019 [https://doi.org/10.1183/23120541.00219-2019]. 


\begin{tabular}{|c|c|c|c|c|c|}
\hline Interviews and focus groups & Individuals & Male & Female & $\begin{array}{l}\text { Ethnicity } \\
\text { WB }\end{array}$ & $\begin{array}{l}\text { Ethnicity } \\
\text { SA }\end{array}$ \\
\hline \multicolumn{6}{|l|}{ Patients } \\
\hline Patients not referred to PR & 7 & 4 & 3 & 4 & 3 \\
\hline Patients declined PR & 7 & 3 & 4 & 6 & 1 \\
\hline Patients accepted an offer of PR & 28 & 16 & 12 & 25 & 3 \\
\hline \multicolumn{6}{|l|}{ Professionals } \\
\hline PR providers & 12 & 1 & 11 & & \\
\hline GPs & 12 & 9 & 3 & & \\
\hline Nurses & 8 & 0 & 8 & & \\
\hline Commissioners & 6 & 1 & 5 & & \\
\hline Online survey & Individuals & \multicolumn{2}{|c|}{ Providers } & \multicolumn{2}{|c|}{ Provider response rate } \\
\hline PR provider survey, EoE & 16 & \multicolumn{2}{|r|}{14} & \multicolumn{2}{|c|}{$70.0 \%$} \\
\hline $\begin{array}{l}\text { PR provider survey, remainder of England } \\
\text { and Wales }\end{array}$ & 56 & \multicolumn{2}{|r|}{46} & \multicolumn{2}{|c|}{$25.7 \%$} \\
\hline GP practice survey, EoE & $112^{\#}$ & \multicolumn{2}{|r|}{107} & \multicolumn{2}{|c|}{$25.8 \%$} \\
\hline
\end{tabular}

Those who accepted a PR offer felt unable to cope with their condition and felt what they were doing was insufficient. They wanted support from healthcare professionals (HCPs) and from others in a similar situation. They saw benefit in exercise, believed they could do it and could get to the class. South Asian patients placed high value on following healthcare advice. Patients who declined PR were satisfied with their care or how they were coping, or had lost hope of their condition improving. They had little understanding of PR or felt their breathlessness made exercise impossible.

I just thought, what's the point? I'm getting out of breath just walking from the bus stop... I said, "What are we doing [in PR]?" She said, "You'll be walking on treadmills and doing step ups and things like that" and I said, "It does me in just walking and going upstairs", so I can't see much point in it.

Patient PD6

Some were anxious about joining a group, feared being forced to quit smoking or felt overburdened by appointments and commitments. Reliance on family for transport was a barrier reported by South Asian patients.

Survey and qualitative research showed GPs and nurses valued PR highly but nurses led referrals and reported better understanding of PR. In survey responses, nurses felt more prepared than GPs to make referrals (61 out of $68(89.7 \%)$ versus 19 out of $41(47.5 \%), \mathrm{p}<0.001)$ but commented that they lacked support from GPs in reinforcing PR discussions with patients. Nurses and GPs with specialist respiratory skills were most confident to refer. Only a minority of respondents in primary care felt there was sufficient training on who to refer (43 out of $112,38.4 \%$ ) or how to refer (40 out of $112,35.7 \%$ ). Free-text and qualitative data showed varied awareness of referral criteria and lack of protocols leading to variation in referral practice.

It doesn't look like it's embedded in our practice protocol. We don't have a fixed way of how we do this and how we pick people up.

HCP GP7

Some clinicians rarely referred while one PR provider described being inundated by referrals. PR was often offered later in the disease course when patients felt less able to exercise. Poor continuity of GP-patient relationships and limited consultation time made it harder to discuss PR in a meaningful way.

Increasingly, continuity of care is broken, so you see a patient who you're meeting for the first or second time and the conversation is very different to a patient you've known the last 10 or 15 years. 


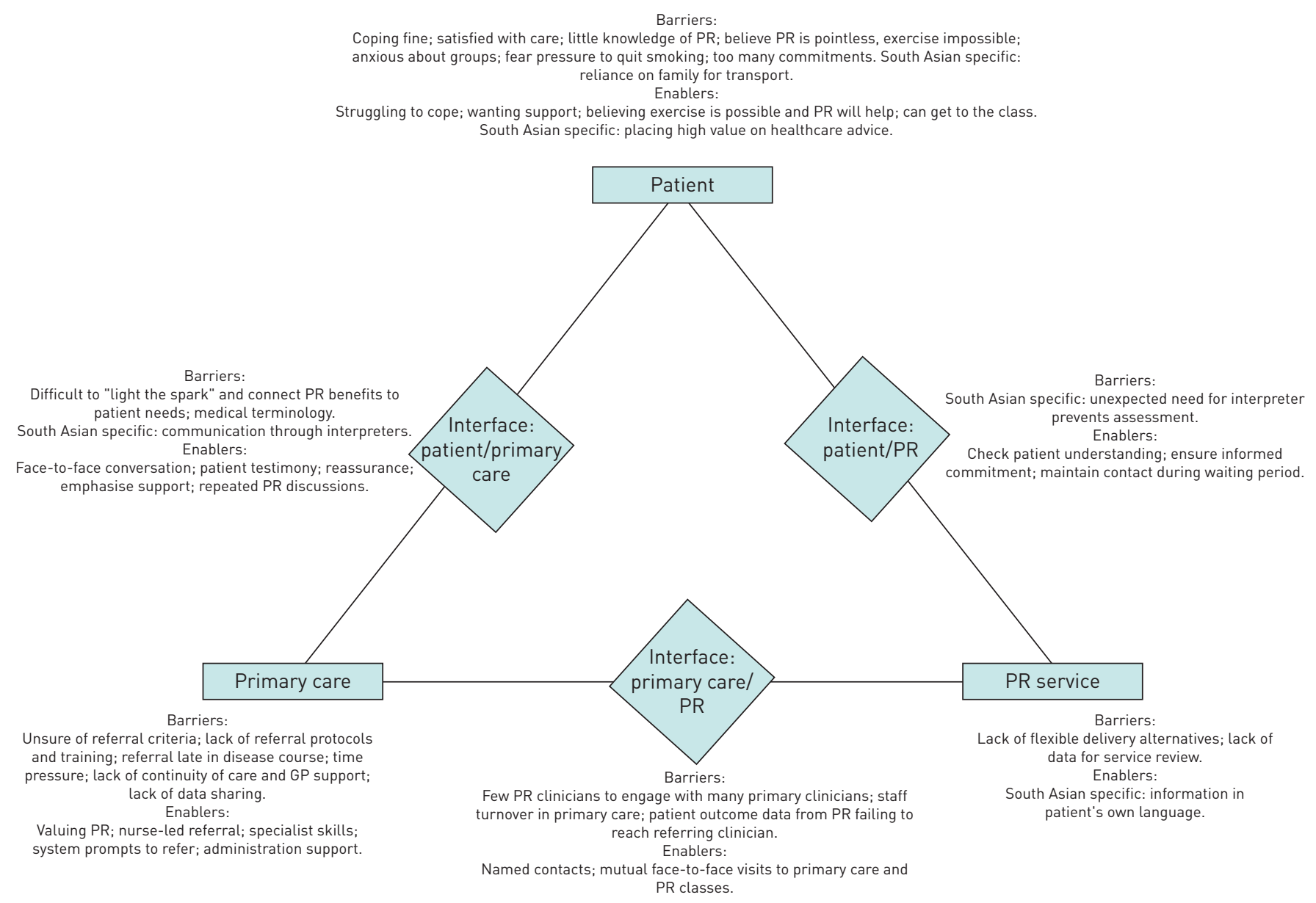

FIGURE 1 Barriers and enablers to pulmonary rehabilitation referral and uptake. PR: pulmonary rehabilitation; GP: general practitioner.

Clinicians wanted to share learning but time was a barrier. System referral prompts and administrative support helped. Sharing referral data across practices or through commissioning groups was limited but highly valued where it occurred.

Some services lacked flexible delivery alternatives that might increase access and not all collected data that could help them to review and improve. Information in South Asian languages was an enabler when available.

Conversations in primary care were vital enablers to exploring PR beliefs. The challenge for clinicians was "lighting the spark", helping patients to connect PR benefits to their own situation and making it sound appealing. This was helped by patient testimonies, presenting PR as a necessary treatment, providing reassurance, and emphasising the fun and support provided at $\mathrm{PR}$.

One of the important things is if whoever is referring, can say, "Once you've started this you've got the whole of the rehab team at your back." That wasn't pointed out to me at the time.

Patient BFG1

The words "pulmonary" and "rehabilitation" were not well understood, and had negative connotations. It helped to develop the conversation over multiple appointments. Some South Asian patients needed an interpreter and some, particularly women, attended appointments with a relative as interpreter; for clinicians, this made it hard to pick up emotional cues in the conversation or be sure of its content.

PR providers checked patient understanding following referral to ensure informed commitment and kept in contact to maintain engagement while the patient was waiting to start. One provider described South Asian patients disengaging from the service when their need for an interpreter at the assessment had not been communicated to the service in advance. 
Face-to-face contacts (e.g. PR visits to practices and GP/nurse visits to PR classes) supported communication, broadened understanding and encouraged referral, but occurred infrequently. PR providers, who were fewer in number, found it challenging to manage relationships with a greater number of GPs and nurses. Staff turnover in primary care disrupted relationships. In the survey, 55 out of $60 \mathrm{PR}$ providers sent patient reports to referrers but many referrers reported not seeing such feedback which, for them, would reinforce referral.

I have not seen a letter recently saying "this patient successfully completed their pulmonary rehab"... They might be sending letters but because there's no action needed from a GP, the letters get filed by the summarisers in the surgery. Or it might be that they don't send a letter."

HCP GP11

Use of NPT and BoT highlights the preconditions of normalisation that need addressing most urgently. Patient barriers could be overcome by face-to-face iterative conversations to encourage understanding of $\mathrm{PR}$ as a positive treatment, taking into account the patient's understanding of their condition(s) and treatments, and the emotional or practical limitations on their capacity for action. This will help the patient make sense of PR, value it and be able to act on their understanding. Such conversations could be challenging for clinicians. Communication between primary care and PR providers was also challenging and sometimes disjointed, which prevented mutual learning. Language barriers in consultations with South Asian patients suggested a healthcare system struggling to adapt to the needs of a minority cultural group. Among clinicians, key barriers to referral reflected NPT constructs of coherence and collective action, i.e. lack of knowledge and operationalising of referrals, rather than lack of value assigned to PR by clinicians or motivation to improve. Interventions are likely to provide most benefit if targeted at nurses, and need to span organisational boundaries to overcome silo working and complement practical solutions [8].

These themes are consistent with the review by MiLner et al. [9], who described low PR knowledge and awareness and difficulties achieving patient behaviour change as barriers to referral. They also reported that lack of belief in the benefits of PR was a barrier to referral. We did not find this, perhaps reflecting a positive bias towards PR in our sample.

Strengths of this study include triangulation of data from different sources and data saturation. Although a low response rate to the primary care survey is a limitation, the themes identified in free text responses were consistent with the qualitative research. While the sample included a relatively smaller number of South Asian patients, themes relating to language, family and gender, specific to this group, were consistent across interviews with these patients.

We identified barriers and enablers to PR referral and uptake in primary care that can inform evidence-based interventions to increase referral and uptake. Improvement requires more effective co-ordination along the COPD pathway.

Frances Early ${ }^{1}$, Patricia Mary Wilson ${ }^{2}$, Christi Deaton ${ }^{3}$, Ian Wellwood ${ }^{3}$, Hena Wali Haque ${ }^{1}$, Sarah Emma Fox ${ }^{4}$, Azka Yousaf ${ }^{4}$, Oliver D. Meysner ${ }^{4}$, James R. Ward ${ }^{5}$, Sally J. Singh ${ }^{6}$ and Jonathan Paul Fuld ${ }^{1}$

${ }^{1}$ Dept of Respiratory Medicine, Cambridge University Hospitals NHS Foundation Trust, Addenbrooke's Hospital, Cambridge, UK. ${ }^{2}$ Centre for Health Services Studies, University of Kent, Canterbury, UK. ${ }^{3}$ Clinical Nursing Research Group, Primary Care Unit, Institute of Public Health, University of Cambridge, Cambridge, UK. ${ }^{4}$ School of Clinical Medicine, University of Cambridge, Cambridge, UK. ${ }^{5}$ Engineering Design Centre, University of Cambridge, UK. ${ }^{6}$ Dept of Respiratory Sciences, University of Leicester, Leicester, UK.

Correspondence: Frances Early, Dept of Respiratory Medicine, Box 146, Cambridge University Hospitals NHS Foundation Trust, Addenbrooke's Hospital, Cambridge Biomedical Campus, Hills Road, Cambridge, CB2 0QQ, UK. E-mail: Frances.early@addenbrookes.nhs.uk

Received: 26 Aug 2019 | Accepted after revision: 11 Dec 2019

Acknowledgements: The authors thank the patients and their families who took part in this study, and those who contributed to the PPI work that supported the design and development of the study. The following were members of the study steering committee with project oversight of the study: Ruth Barlow (Provide Community Interest Company), Lianne Jongepier (NHS North East Essex Clinical Commissioning Group), Mike McKevitt (British Lung Foundation), Sally Singh (University Hospitals of Leicester NHS Trust) and Jenna Stockwell (British Lung Foundation). We also thank the Clinical Research Networks Eastern and Noclor North Thames who supported recruitment in primary care. Delivery of this work was supported by the Cambridge Biomedical Research Centre. 
Conflict of interest: F. Early reports grants from the National Institute for Health Research during the conduct of the study. P.M. Wilson has nothing to disclose. C. Deaton reports grants from the National Institute for Health Research during the conduct of the study. I. Wellwood reports Research for Patient Benefit grant PB-PG-1215-20034 from the National Institute of Health Research (NIHR) UK during the conduct of the study. H.W. Haque has nothing to disclose. S.E. Fox has nothing to disclose. A. Yousaf has nothing to disclose. O.D. Meysner has nothing to disclose. J.R. Ward reports grants from the National Institute for Health Research during the conduct of the study; and grants from the National Institute for Health Research and the University of Cambridge outside the submitted work. S.J. Singh has nothing to disclose. J.P. Fuld reports an NIHR Research for Patient Benefit grant during the conduct of the study.

Support statement: This paper presents independent research funded by the National Institute for Health Research (NIHR) under its Research for Patient Benefit (RfPB) Programme (grant reference number PB-PG-1215-20034). The views expressed are those of the authors and not necessarily those of the NHS, the NIHR or the Department of Health. Funding information for this article has been deposited with the Crossref Funder Registry.

\section{References}

1 McCarthy B, Casey D, Devane D, et al. Pulmonary rehabilitation for chronic obstructive pulmonary disease. Cochrane Database Syst Rev 2015; 2: CD003793,

2 Steiner M, Holzhauer-Barrie J, Lowe D, et al. Pulmonary Rehabilitation: Time to Breathe Better. London, Royal College of Physicians, 2015.

3 Early F, Wilson P, Deaton C, et al. Developing an intervention to increase REferral and uptake TO pulmonary REhabilitation in primary care in patients with chronic obstructive pulmonary disease (the REsTORE study): mixed methods study protocol. BMJ Open 2019; 9: e024806.

4 May CR, Finch T. Implementing, embedding and integrating practices: an outline of normalization process theory. Sociology 2009; 43: 535-554.

5 May CR, Eton DT, Boehmer K, et al. Rethinking the patient: using burden of treatment theory to understand the changing dynamics of illness. BMC Health Serv Res 2014; 14: 281.

6 Ritchie J, Lewis J, McNaughton Nicholls C, et al. Qualitative research practice: a guide for social science students and researchers. 2nd Edn. London, Sage, 2014.

7 Saunders B, Sim J, Kingstone T, et al. Saturation in qualitative research: exploring its conceptualization and operationalization. Qual Quant 2018; 52: 1893-1907.

8 Cox NS, Oliveira CC, Lahham A, et al. Pulmonary rehabilitation referral and participation are commonly influenced by environment, knowledge, and beliefs about consequences: a systematic review using the Theoretical Domains Framework. J Physiother 2017; 63: 84-93.

9 Milner SC, Boruff JT, Beaurepaire C, et al. Rate of, and barriers and enablers to, pulmonary rehabilitation referral in COPD: A systematic scoping review. Respir Med 2018; 137: 103-114. 